\section{Author index (Volume 14)}

A

Abbas, G., see Azam, M.

Abbas, G., see Khan, $\mathrm{S}$.

Abdaoui, M., see Fraj, N. B.

Abdaoui, M., see Nizar, B. F.

Abdulwahhab, M. A. \& Jhangeer, A., Symmetries and generalized higher order conserved vectors of the wave equation on

Bianchi I spacetime

Abebe, A., see Ntahompagaze, J.

Abramov, V., Matrix 3-Lie superalgebras and BRST supersymmetry

Abtahi, S. N., see Mazharimousavi, S. H.

Addazi, A., Chaotic instantons in scalar field theory

Addazi, A., More about the instanton/soliton/kink correspondence

Ahmad, S., see Jawad, A.

Ahsan, Z. \& Ali, M., Curvature tensor for the spacetime of general relativity

Aksoy, E., see Akyol, M. A.
9 (2017) 1750120

$2(2017) 1750025$

12 (2017) 1750180

2 (2017) 1750022

2 (2017) 1750028

7 (2017) 1750107

11 (2017) 1750160

4 (2017) 1750062

10 (2017) 1750135

1 (2017) 1750012

6 (2017) 1750088

5 (2017) 1750078

5 (2017) 1750074
Aktaş, B., see

Durmaz, O.

Akyol, M. A., Sarı, R. \& Aksoy, E., Semi-invariant $\xi^{\perp}$-Riemannian submersions from almost contact metric manifolds

Akyol, M. A., Conformal semi-slant submersions

Alasfar, L., see Alsaleh, S.

Ali, A. F., see Capozziello, S.

Ali, A. F., see Sepehri, A.

Ali, A. \& Ozel, C., Geometry of warped product pointwise semi-slant submanifolds of cosymplectic manifolds and its applications www.worldscientific.com

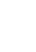

Ali, M., see Ahsan, Z.

3 (2017) 1750042

5 (2017) 1750078

Alsaleh, S. \& Alasfar, L., $E R=E P R$ and non-perturbative action integrals for quantum gravity

Arabzadeh, S. \& Kaviani, K., Multiverse effects on the $C M B$ angular correlation function in the framework of $N C G$ 
Ares, F., Esteve, J.

G., Falceto, F. \&

De Queiroz, A. R., Complex geometry in the

entanglement

entropy of

fermionic chains

Armakan, A. R. \&

Farhangdoost, M.

R., Geometric

aspects of

extensions of

hom-Lie

superalgebras

Aschheim, R.,

Perelman, C. C. \&

Irwin, K., The

search for a

Hamiltonian whose

energy spectrum

coincides with the

Riemann zeta

zeroes

6 (2017) 1750109

Asorey, M., Ibort, A.

\& Spivak, A.,

Admissible

boundary

conditions for

Hamiltonian field

theories

Avendaño-Camacho,

M. \& Vorobiev, Y.,

Deformations of

Poisson structures

on fibered

manifolds and

adiabatic slow-fast systems

Aydin, K., see

Duman, A.

Aydin, S. H., see

Tuğ, G.

Azam, M., Abbas, G., Sumera, S. \&

Nizami, A. R.,

Geodesic structure

of magnetically

charged regular

black hole

9 (2017) 1750120

8 (2017) 1740010

6 (2017) 1750085

8 (2017) 1740006

6 (2017) 1750086

6 (2017) 1750084

5 (2017) 1750069

B

Baffou, E. H.,

Houndjo, M. J. S.

\& Salako, I. G.,
Viscous generalized

Chaplygin gas

interacting with

$f(R, T)$ gravity

4 (2017) 1750051

Bakhshandeh-

Chamazkoti, R.,

Geometry of the

curved traversable

wormholes of

$(3+1)$-dimensional spacetime metric

Balachandran, A. P., Localization in quantum field theory

4 (2017) 1750048

Bamba, K., see

Capozziello, S.

Bartouli, I., see

Basdouri, I.

Basdouri, I.,

Bartouli, I. \&

Lerbet, J.,

Deforming $\mathfrak{h}$-trivial

the Lie algebra

$\operatorname{Vect}\left(S^{1}\right)$ inside the

Lie algebra of pseudodifferential operators $\Psi \mathcal{D O}$

Basdouri, I., Nasri,

E. \& Mechi, H.,

Cohomology of the vector fields Lie

algebras on $\mathbb{R}$

acting on trilinear

differential

operators,

vanishing on

$\mathfrak{a f f}(1)$

Bashir, A., see

Wasay, M. A.

Battista, E.,

Esposito, G., Di

Fiore, L.,

Dell'Agnello, S.,

Simo, J. \& Grado,

A., On solar

system dynamics

in general

relativity

Bayrakdar, T. \&

Ergin, A. A.,

Motion of an

integral curve of a

Hamiltonian

dynamical system
6 (2017) 1750082

10 (2017) 1750150

10 (2017) 1750149 
and the evolution equations in $3 D$

Beesham, A., Gadjagboui, B. B. I. \& Kara, A. H., Classification of a modified de Sitter metric by variational symmetries and conservation laws

Beesham, A., see Sepehri, A.

Bejan, C. L. \& Crasmareanu, M., Parallel second-order tensors on Vaisman manifolds

Bekar, M., see Hathout, F.

Belhaj, A., Bensed, M., Benslimane, Z., Sedra, M. B. \& Segui, A., Qubit and fermionic Fock spaces from type II superstring black holes

Belinchón, J. A. \& Dávila, P., Exact solutions for a scalar-tensor theory through symmetries

Bendjoudi, A. \& Mebarki, N., Loop gravity and Schwarzschild spacetime

Bendjoudi, A. \& Mebarki, N., The quantum polyhedra and the volume spectrum

9 (2017) 1750125

Bennai, M., see Essammouni, K.

Bensed, M., see Belhaj, A.

Benslimane, Z., see Belhaj, A.

6 (2017) 1750087

7 (2017) 1750104

4 (2017) 1750055

7 (2017) 1750100

6 (2017) 1750087

6 (2017) 1750087

Bhandari, P., see Haldar, S.

11 (2017) 1750159
Bhattacharyya, A., see Pahan, S.

4 (2017) 1750050

Bishi, B. K., Pacif, S. K. J., Sahoo, P. K. \& Singh, G. P., LRS Bianchi type-I cosmological model with constant deceleration parameter in $f(R, T)$ gravity

Bishi, B. K., see Sahoo, P. K.

Biswas, I., Moduli spaces of bundles on an abelian variety

Boujelben, J., see Hamza, R.

Boumali, A., see Sargolzaeipor, S.

Bozzetti, C. \& Medori, C., Almost complex manifolds with

non-degenerate torsion

Brevik, I. \&

Timoshkin, A. V., Dissipative universe-inflation with soft singularity

Brevik, I., Elizalde, E., Odintsov, S. D. \& Timoshkin, A. $\mathrm{V}$. , Inflationary universe in terms of a van der Waals viscous fluid

Briscese, F. \& Pucheu, M. L., Palatini formulation of non-local gravity

\section{C}

Cadeddu, L. \& Farina, M. A., A short note on the mean exit time of the Brownian motion Canfes, E. Ö., see Gül, $\dot{\mathrm{I}}$.
2 (2017) 1750030

1 (2017) 1750002

3 (2017) 1750033 4 (2017) 1750061

11 (2017) 1750158

6 (2017) 1750097

7 (2017) 1750112

12 (2017) 1750185
12 (2017) 1750173

9 (2017) 1750122 
Capozziello, S., De

Falco, V. \&

Pincak, R.,

Torsion in Bianchi

IX cosmology

Capozziello, S.,

Saridakis, E. N.,

Bamba, K.,

Sepehri, A.,

Rahaman, F., Ali,

A. F., Pincak, R.

\& Pradhan, A.,

Cosmic space and

Pauli exclusion

principle in a

system of

M0-branes

Capozziello, S., see

Sepehri, A.

Cariñena, J. F.,

Clemente-Gallardo,

J., Jover-Galtier, J.

A. \& Marmo, G.,

Tangent bundle

geometry from

dynamics:

Application to the Kepler problem

Chakraborty, S., see Haldar, S.

Chanda, S., Gibbons, G. W. \& Guha, P., Jacobi-Maupertuis metric and Kepler equation

12 (2017) 1750186

6 (2017) 1750095

7 (2017) 1750099

3 (2017) 1750047

11 (2017) 1750159

7 (2017) 1730002

Chanda, S., Guha, P.

\& Roychowdhury,

R., Schwarzschild

instanton in

emergent gravity

Chattopadhyay, S., A

study on the

bouncing behavior

of modified

Chaplygin gas in

presence of bulk

viscosity and its

consequences in

the modified

gravity framework

Chattopadhyay, S.,

Interacting

modified Chaplygin

gas in $f(T)$ gravity

framework and

1 (2017) 1750006 analysis of its

stability against

gravitational

perturbation

Chen, B.-Y. \&

Verstraelen, L., A

link between

torse-forming

vector fields and

rotational

hypersurfaces

12 (2017) 1750177

Chen, F., see Yan, Z.-W.

1 (2017) 1750013

Chenaghlou, A., see

Mohammadi, V.

1 (2017) 1750004

Cherif, A. M., Some

results on

harmonic and

bi-harmonic maps

7 (2017) 1750098

Chouikh, A., see

Essammouni, K.

Chung, W. S. \&

Kim, J. Y., On the $q$-deformed

circular imaginary

unit and hyperbolic imaginary unit:

$q$-deformed

rotation in two

dimension and

$q$-deformed special

relativity in $1+1$

dimension

4 (2017) 1750057

Chung, W. S., Bose

description of the

generalized Pauli

spin operators and

para super

symmetry

Ciaglia, F. M., Di

Cosmo, F.,

Laudato, M. \&

Marmo, G.,

Differential

calculus on

manifolds with

boundary

applications

8 (2017) 1740003

Cirilo-Lombardo, D.

J. \& Pervushin, V.

N., Quaternionic

(super) twistors

extensions and

general

superspaces

1 (2017) 1750009 
Cirilo-Lombardo, D.

J.,

Non-Riemannian

generalizations of

the Born-Infeld

model and the

meaning of the

cosmological term

7 (2017) 1750108

Clemente-Gallardo,

J., see Cariñena, J.

F.

3 (2017) 1750047

Clyde, S. W., see Milani, V.

Corda, C., see Licata, I.

Corda, C., see Resconi, G.

Crasmareanu, M., see Bejan, C. L.

\section{D}

Darabi, F., see Gholami, F.

Das, A., Singular reduction of Nambu-Poisson manifolds

Davidov, J., Ul Haq,

A. \& Mushkarov, O., Harmonicity of proper almost complex structures on Walker 4-manifolds

Dávila, P., see Belinchón, J. A.

De, U. C., see Mallick, S.

De, U. C., see Suh, Y. J.

De, U. C., Velimirović, L. \& Mallick, S., On a type of spacetime

De Falco, V., see Capozziello, S.

De Queiroz, A. R., see Ares, F.

del Amor, J.,

Giménez, A. \&

Lucas, P., Integrability aspects of the vortex filament

3 (2017) 1750040

7 (2017) 1750110

2 (2017) 1750023

2 (2017) 1750021

9 (2017) 1750128

6 (2017) 1750094

7 (2017) 1750104

6 (2017) 1750096

9 (2017) 1750119

1 (2017) 1750003

8 (2017) 1740010 equation for

pseudo-null curves

6 (2017) 1750090

Delenda, Y., see

Zaim, S.

10 (2017) 1750141

Dell'Agnello, S., see

Battista, E.

9 (2017) 1750117

Demir, S. \& Tanışlı, M., Spacetime algebra for the reformulation of fluid field equations 5 (2017) 1750075

11 (2017) 1730003

12 (2017) 1750186

Deng, S. \& Li, J., Some cohomogeneity one Einstein-Randers metrics on 4-manifolds 3 (2017) 1750044

Dey, R. \& Ganguli, S., The dimension of the Hilbert space of geometric quantization of vortices on a Riemann surface

Dey, R., Geometric quantization of the Hitchin system

4 (2017) 1750064

Díaz-Marín, H. G., Dirichlet to

Neumann operator for Abelian

Yang-Mills gauge fields

11 (2017) 1750153

di Cosmo, F. \& Zampini, A., Dirac operators on the $\mathrm{S}^{3}$ and $\mathrm{S}^{2}$ spheres

Di Cosmo, F., see Ciaglia, F. M.

Di Fiore, L., see Battista, E.

10 (2017) 1750144

8 (2017) 1740005

8 (2017) 1740003

9 (2017) 1750117

Do, T. \& Prince, G., An intrinsic and exterior form of the Bianchi identities

1 (2017) 1750001

Drachal, K., On b-boundary in differential spaces category

3 (2017) 1750036

Duman, A. \& Aydin, K., Sensitivity of Hurwitz stability of linear differential 
equation systems with constant coefficients

Durmaz, O., Aktaş, B. \& Gündoğan, H., The derivative and tangent operators of a motion in Lorentzian space

Dzhunushaliev, V., Small

nonassociative corrections to the SUSY generators and cosmological constant

E

Eken Meriç, Ş., Kiliç, E. \& Sağiroğlu, Y., Scalar curvature of Lagrangian Riemannian submersions and their harmonicity

Ekmekci, F. N., see Tuğ, G.

El Gomdi, N. \& Messaoud, R., Cohomology of orthosymplectic Lie superalgebra acting on $\lambda$-densities on $\mathbb{R}^{1 \mid n}$

Elamine, N., see Okba, B.

Elizalde, E., see Brevik, I.

Elkhatib, B., see Nashed, G. G. L.

Ergin, A. A., see Bayrakdar, T.

Erman, F., On the number of bound states of point interactions on hyperbolic manifolds

Eshaghi Gordji, M., see Park, C.

Espinoza, M., see Nieto, J. A.
6 (2017) 1750084

4 (2017) 1750058

5 (2017) 1750072

12 (2017) 1750171

5 (2017) 1750069

1 (2017) 1750016

1 (2017) 1750015

12 (2017) 1750185

11 (2017) 1750154

12 (2017) 1750172

1 (2017) 1750011

5 (2017) 1750079

1 (2017) 1750014
Esposito, G., see

Battista, E.

9 (2017) 1750117

Essammouni, K., Chouikh, A., Said, T. \& Bennai, M., niSWAP and NTCP gates realized in a circuit QED system 7 (2017) 1750100

Esteve, J. G., see Ares, F.

8 (2017) 1740010

F

Fabbri, L., Torsion gravity for Dirac fields

3 (2017) 1750037

Fabbri, L., Torsion gravity for Dirac particles

9 (2017) 1750127

Facchi, P. \& Garnero, G., Quantum thermodynamics and canonical typicality 8 (2017) 1740001

Facchi, P., Garnero, G. \& Ligabò, M., Quantum fluctuation relations

Faidi, T., see Khalfoun, H.

Fakhri, H. \& Sayyah-Fard, M., Arik-Coon $q$-oscillator cat states on the noncommutative complex plane $\mathbb{C}_{q^{-1}}$ and their nonclassical properties

Fakhri, H. \&

Sayyah-Fard, M., Nonclassical properties of the Arik-Coon $q^{-1}$-oscillator coherent states on the noncommutative complex plane $\mathbb{C}_{q}$ 8 (2017) 1740002 2 (2017) 1750027 4 (2017) 1750060

Falceto, F., see Ares, F.

11 (2017) 1750165

8 (2017) 1740010

Fang, X.-L., Kim, T.-H. \& Zhang, 
X.-H., Symmetry

and pseudo-

symmetry of

$v$-Yetter-Drinfeld

categories for

Hom-Hopf

algebras

9 (2017) 1750129

Farhangdoost, M. R., see Armakan, A. $\mathrm{R}$.

6 (2017) 1750085

Farina, M. A., see Cadeddu, L.

Ferrara, L., see Marasco, A.

Forghani, S. D., see Mazharimousavi, S. H.

Fraj, N. B., Laraiedh, I. \& Abdaoui, M., Cohomology of $\mathfrak{a f f}(2)$ acting on some modules and deformations

Frønsdal, C., Relativistic thermodynamics, a Lagrangian field theory for general flows including rotation

\section{G}

Gadjagboui, B. B. I., see Beesham, A.

Ganguli, S., see Dey, R.

Garat, A., The new electromagnetic tetrads, infinite tetrad nesting and the non-trivial emergence of complex numbers in real theories of gravitation

Garnero, G., see Facchi, P.

Garnero, G., see Facchi, P.

Ghaffar, A., see Wasay, M. A.

Ghaffary, T., see Parang, Z.

Ghaffary, T., see Sepehri, A.

4 (2017) 1750062

2 (2017) 1750017

9 (2017) 1750132

8 (2017) 1740001

8 (2017) 1740002

9 (2017) 1750130
Ghaffary, T., Comparing production cross-sections for $Q C D$ matter, Higgs boson, neutrino with dark energy in accelerating universe

10 (2017) 1750139

12 (2017) 1750173

11 (2017) 1750151

Gharahbeigi, M. M., see Parang, Z.

11 (2017) 1750163

Gholami, F., Darabi, F. \& Haji-Badali, A., Multiply-warped product metrics and reduction of Einstein equations

2 (2017) 1750021

Ghominejad, M., see Nayeh, S.

4 (2017) 1750053

12 (2017) 1750180

12 (2017) 1750182

10 (2017) 1750144

Ghose-Choudhury, A., Guha, P., Paliathanasis, A. \& Leach, P. G. L., Noetherian symmetries of noncentral forces with drag term

2 (2017) 1750018

Gibbons, G. W., see Chanda, S.

7 (2017) 1730002

Giménez, A., see del Amor, J.

6 (2017) 1750090

Grado, A., see

Battista, E.

Guelmamene, H., see Zaim, S.

9 (2017) 1750117

10 (2017) 1750141

Guha, P., see Chanda, S.

Guha, P., see Chanda, S.

Guha, P., see Ghose-Choudhury,

10 (2017) 1750149

11 (2017) 1750163
A.

Gül, İ. \& Canfes, E. Ö., $O n$ quasi-Einstein Weyl manifolds

Gündoğan, H., see Durmaz, O.

Gupta, M. K. \& Yadav, C. K., Jacobi stability analysis of
2 (2017) 1750018

1 (2017) 1750006

7 (2017) 1730002

9 (2017) 1750122

4 (2017) 1750058 
modified Chua

circuit system

Gur, S., see Şenyurt, $\mathrm{S}$.

6 (2017) 1750089

9 (2017) 1750118

Gürbüz, N.,

Anholonomy according to three

formulations of non-null curve evolution

12 (2017) 1750175

\section{H}

Haji-Badali, A., see Gholami, F.

Haldar, S., Bhandari, P. \& Chakraborty, S., $A$ thermodynamical analysis of the inhomogeneous FLRW type model: Redefined BekensteinHawking system

Halilsoy, M., see Mazharimousavi, S. H.

Hamza, R., Selmi, Z. \& Boujelben, J., Differential operators on the supercircle $S^{1 \mid 2}$ and symbol map

Hamza, R., see Nizar, B. F.

Han, J.-M., see Yan, Z.-W.

Hansen, A. K. \& Sutlu, S. S., $A$ minimal realization for affine control systems on connected Lie groups

Hassanabadi, H., see Sargolzaeipor, S.

Hathout, F., Bekar, M. \& Yayli, Y., Ruled surfaces and tangent bundle of unit 2-sphere

Hejazi, S. R., see

Rashidi, S.

2 (2017) 1750021

11 (2017) 1750159

11 (2017) 1750155

1 (2017) 1750002

2 (2017) 1750022

1 (2017) 1750013

9 (2017) 1750126

7 (2017) 1750112

10 (2017) 1750145

12 (2017) 1750170
Hejazi, S. R., see Rashidi, S.

6 (2017) 1750083

Holweck, F., see

Saniga, M.

5 (2017) 1750080

Houndjo, M. J. S., see Baffou, E. H.

Huang, R., Song, T. \& Li, C., Gauge transformations of constrained discrete modified KP systems with self-consistent sources

4 (2017) 1750052

Hussain, T., see

Khan, S.

3 (2017) 1750043

I

Ibort, A., see Asorey, $\mathrm{M}$.

8 (2017) 1740006

Ikiz, H., see Karacan, M. K.

5 (2017) 1750076

İlarslan, K., see Uçum, A.

10 (2017) 1750143

Ilyas, A., see Jawad, A.

6 (2017) 1750088

Irwin, K., see

Aschheim, R.

6 (2017) 1750109

$\mathbf{J}$

Jain, V., Rani, R., Kumar, R. \& Nagaich, R. K., Some characterization

theorems on

holomorphic

sectional curvature of GCR-lightlike submanifolds

3 (2017) 1750034

Jamal, S., Potentials and point symmetries of Klein-Gordon equations in space-time homogenous Gödel-type metrics 5 (2017) 1750070

Jawad, A., Ilyas, A. \& Ahmad, S., Shaft potential inspired warm inflation Jhangeer, A., see Abdulwahhab, M. A. 2 (2017) 1750028 
Joharinad, P., Warped product Finsler manifolds from Hamiltonian point of view

Jover-Galtier, J. A., see Cariñena, J. F.

Jung, S. D., see Liu, $\mathrm{H}$.

Jusufi, K., Deflection angle of light by wormholes using the Gauss-Bonnet theorem

Jusufi, K., Quantum effects on the deflection of light and the

Gauss-Bonnet theorem

\section{K}

Kanatchikov, I. V., On the spectrum of $D W$ Hamiltonian of quantum SU(2) gauge field

Kara, A. H., see Beesham, A.

Karacan, M. K., Yuksel, N. \& Ikiz, H., On ruled surface in 3-dimensional almost contact metric manifold

Karakus, F., see Uçar, A.

Kassandrov, V. V. \& Rizcallah, J. A., Maxwell, Yang-Mills, Weyl and eikonal fields defined by any null shear-free congruence

Kaviani, K., see Arabzadeh, S.

Kempf, A., see Panine, $\mathrm{M}$.

Keskin, O. \& Yayli, Y., An application of $N$-Bishop frame to spherical images
2 (2017) 1750029

3 (2017) 1750047

5 (2017) 1750066

12 (2017) 1750179

10 (2017) 1750137

9 (2017) 1750123

12 (2017) 1750182

5 (2017) 1750076

9 (2017) 1750131

2 (2017) 1750031

4 (2017) 1750054

11 (2017) 1750157 for direction curves

11 (2017) 1750162

Khalfoun, H. \& Faidi, T., On the $\mathfrak{a f f}(m \mid 1)$-relative cohomology of the orthosymplectic superalgebra $\mathfrak{o s p}(m \mid 2)$ and $\mathfrak{a} \mathfrak{f} \mathfrak{f}(m \mid 1)$-trivial deformation

2 (2017) 1750027

Khalfoun, H., $\mathfrak{a f f}(1 \mid 1)$-Relative cohomology on $\mathbb{R}^{1 \mid 1}$

12 (2017) 1750174

Khan, G. A., see Khan, S.

Khan, S., Hussain, T. \& Khan, G. A., Teleparallel conformal Killing vector fields of LRS Bianchi type $V$ spacetimes in teleparallel gravity

Khan, S., Shah, H. \& Abbas, G., Five-dimensional spherical gravitational collapse of anisotropic fluid with cosmological constant

Khosropour, B., Lorentz force and ponderomotive force in the presence of a minimal length

Khurshudyan, M., Phase space analysis in a model of $f(T)$ gravity with nonlinear sign changeable interactions

Kiliç, E., see Eken Meriç, Ş.

Kim, J. Y., see

Chung, W. S.

Kim, T.-H., see Fang, X.-L.

Kiṣi, İ. \& Öztürk, G., $A$ new approach to
2 (2017) 1750025

3 (2017) 1750043

3 (2017) 1750043

1 (2017) 1750005
12 (2017) 1750171

4 (2017) 1750057

9 (2017) 1750129 
canal surface with parallel transport frame

Koch, B., see Wasay, M. A.

2 (2017) 1750026

Komal, A., see Shamir, M. F.

Kumar, R., see Jain, V.

Kumar, S., Some characterizations on minimal lightlike submanifolds

Küpeli Erken, İ. \& Murathan, C., A study of three-dimensional paracontact $(\tilde{\kappa}, \tilde{\mu}, \tilde{\nu})$-spaces

L

Laraiedh, I., see Fraj, N. B.

Laudato, M., see Ciaglia, F. M.

Leach, P. G. L., see Ghose-Choudhury, A.

Lerbet, J., see Basdouri, I.

Li, C., see Huang, R. Li, J., see Deng, S.

Licata, I., Moradpour, H. \& Corda, C., The commutator algebra of covariant derivative as general framework for extended gravity. The Rastall theory case and the role of the torsion

Licata, I., see Resconi, G.

Ligabò, M., see Facchi, P.

Liu, H. \& Jung, S. D., Structures and properties of null scroll in Minkowski 3-space
11 (2017) 1730003

10 (2017) 1750149

12 (2017) 1750169

3 (2017) 1750034

7 (2017) 1750103

7 (2017) 1750106

12 (2017) 1750180

8 (2017) 1740003

2 (2017) 1750018

6 (2017) 1750082

4 (2017) 1750052

3 (2017) 1750044

7 (2017) 1750110

8 (2017) 1740002

5 (2017) 1750066
Liu, J., Sheng, Y. \&

Wang, C., Omni

$n$-Lie algebras and linearization of higher analogues of Courant algebroids 7 (2017) 1750113

Liu, T.-R., see Yan, Z.-W.

1 (2017) 1750013

Lucas, P., see del Amor, J.

6 (2017) 1750090

\section{M}

Mağden, A., Yılmaz, S. \& Yasin

Ünlütürk

Characterizations

of special time-like curves in

Lorentzian plane $\mathbb{L}^{2}$

10 (2017) 1750140

Makarenko, A. N. \& Myagky, A. N., The asymptotic behavior of bouncing cosmological models in $F(\mathcal{G})$ gravity theory

Maleki, M., see Rajabi, $\mathrm{T}$.

Mallick, S., Yildiz, A. \& De, U. C., Characterizations of mixed quasi-Einstein manifolds

6 (2017) 1750096

Mallick, S., see De, U. C.

1 (2017) 1750003

Mandal, R., see Sarkar, K.

3 (2017) 1750038

Mansourbeigi, S. M. H., see Milani, V.

Mantica, C. A. \& Molinari, L. G., Generalized Robertson-Walker spacetimes - A survey

Mantica, C. A. \&

Shenawy, S., Einstein-like warped product manifolds

11 (2017) 1750166

Mantica, C. A., see Suh, Y. J.
3 (2017) 1730001

3 (2017) 1750040

9 (2017) 1750119 
Marasco, A., Ferrara, L. \& Romano, A., Modeling eutrophic lakes: From mass balance laws to ordinary differential equations

Marmo, G., see Cariñena, J. F.

Marmo, G., see Ciaglia, F. M.

Massamba, F. \& Ssekajja, S., A note on quasi-generalized CR-lightlike geometry in indefinite nearly $\mu$-Sasakian manifolds

Maurya, D. C., Zia, R. \& Pradhan, A., Dark energy models in LRS Bianchi type-II space-time in the new perspective of time-dependent deceleration parameter

Mazharimousavi, S. H. \& Halilsoy, M. Morse simulation of the global monopole equation in flat spacetime

Mazharimousavi, S. H., Forghani, S. D. \& Abtahi, S. N., Generalized Monge gauge

Mbonye, M., see Ntahompagaze, J.

Mebarki, N., see Bendjoudi, A.

Mebarki, N., see Bendjoudi, A.

Mechi, H., see Basdouri, I.

Medori, C., see Bozzetti, C.

Messaoud, R., see El Gomdi, N.
11 (2017) 1750151

3 (2017) 1750047

8 (2017) 1740003

3 (2017) 1750045

5 (2017) 1750077

11 (2017) 1750155

4 (2017) 1750062

7 (2017) 1750107

4 (2017) 1750055

9 (2017) 1750125

10 (2017) 1750150

3 (2017) 1750033

1 (2017) 1750016
Milani, V.,

Mansourbeigi, S.

M. H. \& Clyde, S.

W., Geometry of

physical systems

on quantized

spaces

3 (2017) 1750040

Mishra, B., Sahoo, P.

K. \& Ray, P. P.,

Accelerating dark

energy

cosmological model

in two fluids with

hybrid scale factor

Modanese, G.,

Ultra-light and

strong: The

massless harmonic

oscillator and its

singular path

integral

1 (2017) 1750010

Mohammadi, V. \&

Chenaghlou, A.,

Dirac equation

with anisotropic

oscillator, quantum $E 3^{\prime}$ and Holt superintegrable potentials and relativistic generalized Yang-Coulomb monopole system

Molinari, L. G., see Mantica, C. A.

Mollah, M. R., see Singh, K. P.

9 (2017) 1750124

Moradpour, H., see Licata, I.

Murathan, C., see Küpeli Erken, İ.

Mushkarov, O., see Davidov, J.

1 (2017) 1750004

3 (2017) 1730001

4 (2017) 1750063

11 (2017) 1730003

7 (2017) 1750106

6 (2017) 1750094

Myagky, A. N., see Makarenko, A. N.

Myrzakul, A. \& Myrzakulov, R., Integrable geometric flows of interacting curves/surfaces, multilayer spin systems and the vector nonlinear 
Schrödinger equation

Myrzakul, A. \& Myrzakulov, R., Integrable motion of two interacting curves, spin systems and the Manakov system

Myrzakul, S., see Pacif, S. K. J.

Myrzakulov, R., see Myrzakul, A.

Myrzakulov, R., see Myrzakul, A.

Myrzakulov, R., see Pacif, S. K. J.

Myrzakulov, R., see Samanta, G. C.

Myrzakulov, R., see Sebastiani, L.

$\mathbf{N}$

Nadjafikhah, M., see Rahimian, M.

Nagaich, R. K., see Jain, V.

Nashed, G. G. L. \& Elkhatib, B.,

Fixing redundant degrees of teleparallel equivalent of general relativity

Nashed, G. G. L., Charged three-dimensions black holes in Weitzenböck geometry

Nasri, E., see Basdouri, I.

Nayeh, S. \& Ghominejad, M., Weyl static axially symmetric field equations in modified $f(T)$ gravity

Nešović, E., On geometric interpretation of pseudo-angle in Minkowski plane
10 (2017) 1750136

7 (2017) 1750115

7 (2017) 1750111

10 (2017) 1750136

7 (2017) 1750115

7 (2017) 1750111

12 (2017) 1750183

6 (2017) 1750093

3 (2017) 1750046

3 (2017) 1750034

11 (2017) 1750154

7 (2017) 1750105

10 (2017) 1750150

4 (2017) 1750053

5 (2017) 1750068
Nieto, J. A. \&

Espinoza, M., Dirac equation in four time and four space dimensions

1 (2017) 1750014

Nizami, A. R., see Azam, M.

Nizar, B. F.,

Abdaoui, M. \& Hamza, R., On $\mathfrak{o s p}(1 \mid 2)$-relative cohomology of the Lie superalgebra of contact vector fields on $\mathbb{R}^{1 \mid 1}$

Nozari, K., see

Saghafi, S.

2 (2017) 1750022

11 (2017) 1750164

Ntahompagaze, J., Abebe, A. \& Mbonye, M., On $f(R)$ gravity in scalar-tensor theories

9 (2017) 1750120

7 (2017) 1750107

O

Odintsov, S. D., see Brevik, I.

12 (2017) 1750185

Oiwa, S. \& Yajima, T., Jacobi stability analysis and chaotic behavior of nonlinear double pendulum

12 (2017) 1750176

Okba, B. \& Elamine, N., $\mathfrak{s l}(2, \mathbb{R})$-invariant 3-ary differential operators and cohomology of $\mathfrak{s l}(2, \mathbb{R})$ acting on 3-ary differential operators

1 (2017) 1750015

Olmo, G. J., see Sepehri, A.

11 (2017) 1750167

Özdemir, Z., see Tuğ, $\mathrm{G}$.

5 (2017) 1750069

Ozel, C., see Ali, A.

Özer, H. T., Casimir

3 (2017) 1750042

$\mathcal{W} \mathcal{A}_{N}$ algebras as the truncated $\mathcal{W}_{\infty}$ algebra

9 (2017) 1750121

Öztürk, G., see Kiși, I.

2 (2017) 1750026 
$\mathbf{P}$

Pacif, S. K. J.,

Myrzakulov, R. \&

Myrzakul, S.,

Reconstruction of

cosmic history

from a simple

parametrization of H

Pacif, S. K. J., see Bishi, B. K.

Pahan, S., Pal, B. \& Bhattacharyya, A., On Einstein warped products with a quarter-symmetric connection

Pal, B., see Pahan, S.

Pal, P., see Suh, Y. J.

Paliathanasis, A., see Ghose-Choudhury, A.

Pandey, M. \& Vaidya, S., Yang-Mills matrix mechanics and quantum phases

Panine, M. \& Kempf, A., A convexity result in the spectral geometry of conformally equivalent metrics on surfaces

Papadopoulos, K., On the possibility of singularities on the ambient boundary

Parang, Z., Ghaffary, T. \& Gharahbeigi, M. M., Effect of elastic constants of liquid crystals in their electro-optical properties

Park, C., Eshaghi Gordji, M. \& Tavakkoli Targhi, A., Comment to "Approximate bihomomorphisms
7 (2017) 1750111

11 (2017) 1750158

4 (2017) 1750050

4 (2017) 1750050

9 (2017) 1750119

2 (2017) 1750018

8 (2017) 1740009

11 (2017) 1750157

12 (2017) 1750184

11 (2017) 1750163 and biderivations in 3-Lie algebras" [Int. J. Geom.

Methods Mod.

Phys. 10 (2013)

1220020]

5 (2017) 1750079

Park, I. Y. Reduction of gravity-matter and $d S$ gravity to hypersurface

Perelman, C. C., see Aschheim, R.

Pérez-Pardo, J. M., Dirac-like operators on the Hilbert space of differential forms on manifolds with boundaries

Pervushin, V. N., see Cirilo-Lombardo, D. J.

Peterka, M. A. \& Sheu, A. J.-L., On noncommutative Levi-Civita connections

Pincak, R., see Capozziello, S.

Pincak, R., see Capozziello, S.

Pincak, R., see Sepehri, A.

Pincak, R., see Sepehri, A.

Pincak, R., see Smotlacha, J.

Pirhadi, V. \& Razavi, A., On the almost quasi-Yamabe solitons

Pracna, P., see Saniga, M.

Pradhan, A., see Capozziello, S.

Pradhan, A., see Maurya, D. C.

Pradhan, A., see Sepehri, A.

Pradhan, A., see Sepehri, A.

Pradhan, P., Circular orbits in the Taub-NUT and
8 (2017) 1740004

1 (2017) 1750009

11 (2017) 1750161

6 (2017) 1750092

6 (2017) 1750109

5 (2017) 1750071

12 (2017) 1750186

6 (2017) 1750095

11 (2017) 1750167

9 (2017) 1750130

9 (2017) 1750116

5 (2017) 1750080

6 (2017) 1750095

5 (2017) 1750077

7 (2017) 1750099

9 (2017) 1750130 
massless

Taub-NUT

spacetime

Prajapati, G. K., see

Tiwari, B.

7 (2017) 1750101

5 (2017) 1750081

Prince, G., see Do, T.

Pucheu, M. L., see

Briscese, F.

\section{$\mathbf{R}$}

Rahaman, F., see Capozziello, S.

Rahaman, F., see Sepehri, A.

Rahaman, F., see Sepehri, A.

Rahimian, M. Toomanian, M. \& Nadjafikhah, M., Approximate symmetry and solutions of the nonlinear Klein-Gordon equation with a small parameter

Rajabi, T., Sadeghzadeh, N. \& Maleki, M., On invariant Finsler metrics under (almost) $\beta$-changes

Rajpoot, S. \& Vacaru, S. I., Nonholonomic jet deformations, exact solutions for modified Ricci soliton and Einstein equations

Rañada, A. F., Tiemblo, A. \& Trueba, J. L., Time evolving potentials for electromagnetic knots

Rani, R., see Jain, V.

Rashidi, S. \& Hejazi, S. R., Analyzing Lie symmetry and constructing
1 (2017) 1750001

2 (2017) 1750019

6 (2017) 1750095

7 (2017) 1750099

9 (2017) 1750130

3 (2017) 1750046

11 (2017) 1750156

2 (2017) 1750032

3 (2017) 1750034 conservation laws for time-fractional Benny-Lin equation

Rashidi, S. \& Hejazi, S. R., Symmetry properties, similarity reduction and exact solutions of fractional

Boussinesq equation

Ray, P. P., see Mishra, B.

Razavi, A., see Pirhadi, V.

Resconi, G., Licata, I. \& Corda, C., Gravity with torsion in non-conservative

Maxwell-like gauge approach

7 (2017) 1750110

Rezaei-Aghdam, A.

\& Sedghi-Ghadim, L., 3-Leibniz bialgebras (3-Lie bialgebras)

6 (2017) 1750083

9 (2017) 1750124

11 (2017) 1750161

Rezaei-Aghdam, A \& Sephid, M., Classification of real low-dimensional Jacobi

(generalized)-Lie bialgebras

Rezaii, M. M., see Shojaee, N.

Rizcallah, J. A., see Kassandrov, V. V. Romano, A., see Marasco, A.

Roychowdhury, R., see Chanda, S.

1 (2017) 1750007

1 (2017) 1750008

2 (2017) 1750031

11 (2017) 1750151

1 (2017) 1750006

$\mathbf{S}$

Sadeghzadeh, N., see Rajabi, T.

11 (2017) 1750156

Saghafi, S. \& Nozari, K., Black hole thermodynamics in Snyder phase space
10 (2017) 1750142 
Sağiroğlu, Y., see

Eken Meriç, Ş.

Șahin, B. \& Șahin,

F., Generalized

almost

para-contact

manifolds

Șahin, F., see Șahin, B.

Sahoo, P. K., Sahoo, P. \& Bishi, B. K., Anisotropic cosmological models in $f(R, T)$ gravity with variable deceleration parameter

Sahoo, P. K., see Bishi, B. K.

Sahoo, P. K., see Mishra, B.

Sahoo, P., see Sahoo, P. K.

Sahraee, M., see Setare, M. R.

Said, T., see Essammouni, K.

Sakaki, M., see Uçum, A.

Salako, I. G., see Baffou, E. H.

Samanta, G. C. \& Myrzakulov, R., Cosmological models constructed by van der Waals fluid approximation and volumetric expansion

Saniga, M., Holweck, F. \& Pracna, P., Veldkamp spaces: From (Dynkin) diagrams to (Pauli) groups

Sanyal, A. K., see Sarkar, K.

Sargolzaeipor, S., Hassanabadi, H. \& Boumali, A. Morse potential of the $q$-deformed in
12 (2017) 1750171

10 (2017) 1750147

10 (2017) 1750147

6 (2017) 1750097

11 (2017) 1750158

9 (2017) 1750124

6 (2017) 1750097

10 (2017) 1750134

7 (2017) 1750100

10 (2017) 1750143

4 (2017) 1750051

12 (2017) 1750183

5 (2017) 1750080

3 (2017) 1750038 the Duffin-

Kemmer-Petiau equation

7 (2017) 1750112

Saridakis, E. N., see Capozziello, S.

6 (2017) 1750095

Sarkar, K., Sk, N., Mandal, R. \& Sanyal, A. K., Canonical formulation of Pais-Uhlenbeck action and resolving the issue of branched

Hamiltonian

3 (2017) 1750038

Sarı, R., see Akyol, M. A.

5 (2017) 1750074

Sayyah-Fard, M., see Fakhri, H.

11 (2017) 1750165

Sayyah-Fard, M., see Fakhri, H.

4 (2017) 1750060

Sebastiani, L. \& Myrzakulov, R., $k$-essence for warm inflation on the brane

6 (2017) 1750093

Sedghi-Ghadim, L., see

Rezaei-Aghdam,

A.

10 (2017) 1750142

Sedra, M. B., see

Belhaj, A.

6 (2017) 1750087

Segui, A., see Belhaj, A.

6 (2017) 1750087

Selmi, Z., see Hamza, $\mathrm{R}$.

1 (2017) 1750002

Şenyurt, S. \& Gur, S., Spacelike surface geometry

Sepehri, A., Pincak, R. \& Olmo, G. J., $M$-theory, graphene-branes and

superconducting wormholes

Sepehri, A., Pradhan, A., Pincak, R., Rahaman, F., Beesham, A. \& Ghaffary, T., Birth of the GUP and its effect on the entropy of the 
universe in

Lie-N-algebra

Sepehri, A.,

Rahaman, F.

Capozziello, S.,

Ali, A. F. \&

Pradhan, A., The

evolution of

Brown-York

quasilocal energy

as due to evolution

of Lovelock gravity

in a system of

M0-branes

Sepehri, A., see

Capozziello, S.

Sepehri, A., A

mathematical

model for DNA

Sephid, M., see

Rezaei-Aghdam, A.

Setare, M. R. \&

Sahraee, M., The

effect of

backreaction on

inflationary

Starobinsky

cosmology

Shah, H., see Khan,

S.

Shamir, M. F. \&

Komal, A., Energy

bounds in $f(R, G)$

gravity with

anisotropic

background

Shenawy, S., see Mantica, C. A.

Sheng, Y., see Liu, J.

Sheu, A. J.-L., see

Peterka, M. A.

Shojaee, N. \& Rezaii,

M. M., On the

conformal scalar

curvature

equations on

Finsler manifolds

Simo, J., see

Battista, E.

Singh, G. P., see

Bishi, B. K.

Singh, K. P. \&

Mollah, M. R.,
9 (2017) 1750130

7 (2017) 1750099

6 (2017) 1750095

11 (2017) 1750152

1 (2017) 1750007

10 (2017) 1750134

2 (2017) 1750025

12 (2017) 1750169

11 (2017) 1750166

7 (2017) 1750113

5 (2017) 1750071

1 (2017) 1750008

9 (2017) 1750117

11 (2017) 1750158
Could the Lyra manifold be the hidden source of the dark energy?

4 (2017) 1750063

Sk, N., see Sarkar,

$\mathrm{K}$.

3 (2017) 1750038

Smotlacha, J. \&

Pincak, R.,

Boundary

conditions and

Green function

approach of the

spin-orbit

interaction in the graphitic nanocone

9 (2017) 1750116

Song, T., see Huang, $\mathrm{R}$.

4 (2017) 1750052

Sorkin, R. D., From

Green function to quantum field

8 (2017) 1740007

Spivak, A., see

Asorey, M.

Ssekajja, S., see

Massamba, F.

Stachura, P., On

Poisson structures

related to

$\kappa$-Poincaré group

Stoica, O. C., The

geometry of warped

product

singularities

8 (2017) 1740006

3 (2017) 1750045

Suh, Y. J., Mantica, C. A., De, U. C. \&

Pal, P., Pseudo

B-symmetric

manifolds

Sumera, S., see

Azam, M.

Sutlu, S. S., see

Hansen, A. K.

Syromyatnikov, A.

G., Electro-gravity

spin density waves

9 (2017) 1750133

2 (2017) 1750024

9 (2017) 1750119

9 (2017) 1750120

9 (2017) 1750126

10 (2017) 1750146

$\mathbf{T}$

Taha, E. H., see

Youssef, N. L.

3 (2017) 1750039

1 (2017) 1750013

Tala, see Yan, Z.-W

Tanışlı, M., see

Demir, S.

5 (2017) 1750075

Tavakkoli Targhi, A., see Park, C.

5 (2017) 1750079

Tiemblo, A., see

Rañada, A. F.
5 (2017) 1750073 
Timoshkin, A. V., see Brevik, I.

Timoshkin, A. V., see Brevik, I.

Tiwari, B. \&

Prajapati, G. K., On generalized

Kropina change of mth root Finsler metric

Toomanian, M., see Rahimian, M.

Torromé, R. G., A second-order differential equation for a point charged particle

Torromé, R. G., On singular generalized Berwald spacetimes and the equivalence principle

Trueba, J. L., see Rañada, A. F.

Tuğ, G., Özdemir, Z., Aydin, S. H. \& Ekmekci, F. N., Accretive growth kinematics in Minkowski 3-space

Tunçer, Y., Vectorial moments of curves in Euclidean 3-space

Turgut Vanli, A. \& Unal, I., Conformal, concircular, quasi-conformal and conharmonic flatness on normal complex contact metric manifolds

\section{U}

Uçar, A., Karakuş, F. \& Yaylı, Y., Generalized Fermi-Walker derivative and non-rotating frame
5 (2017) 1750081

12 (2017) 1750185

4 (2017) 1750061

3 (2017) 1750046

4 (2017) 1750049

6 (2017) 1750091

5 (2017) 1750073

5 (2017) 1750069

2 (2017) 1750020

5 (2017) 1750067

9 (2017) 1750131
Uçum, A., Sakaki, M. \& İlarslan, K., Lorentzian stationary surfaces and bi-null curves in $\mathbb{R}_{2}^{5}$

10 (2017) 1750143

Ul Haq, A., see Davidov, J.

6 (2017) 1750094

Unal, I., see Turgut Vanli, A.

5 (2017) 1750067

V

Vacaru, S. I., see Rajpoot, S.

Vaidya, S., see Pandey, M.

Varshovi, A. A., Gauge fixing invariance and anti-BRST symmetry 11 (2017) 1750168

Veliev, O., On the spectral properties of the Schrödinger operator with a periodic PT-symmetric potential

2 (2017) 1750032

8 (2017) 1740009

Velimirović, L., see

De, U. C.

5 (2017) 1750065

1 (2017) 1750003

Verstraelen, L., see

Chen, B.-Y.

Vorobiev, Y., see

Avendaño-

Camacho, M.

12 (2017) 1750177

6 (2017) 1750086

W

Wang, C., see Liu, J. 7 (2017) 1750113

Wang, Y., An equivariant Kastler-KalauWalze type theorem 4 (2017) 1750056

Wasay, M. A., Bashir, A., Koch, B. \& Ghaffar, A., Geometric description of the Schrödinger equation in $(3 n+1)$-dimensional configuration space

Weng, Z.-H., Spin angular 
momentum of proton spin puzzle in complex

octonion spaces

7 (2017) 1750102

Y

Yadav, C. K., see Gupta, M. K.

Yajima, T., see Oiwa, S.

Yan, Z.-W., Tala, Chen, F., Liu, T.-R. \& Han, J.-M., (2+1)-Dimensional supersymmetric integrable equations

Yasin Ünlütürk, see Mağden, A.

Yayli, Y., see Hathout, F.

Yayli, Y., see Keskin, O.

Yaylı, Y., see Uçar, A.

6 (2017) 1750089

12 (2017) 1750176

1 (2017) 1750013

10 (2017) 1750140

10 (2017) 1750145

11 (2017) 1750162

9 (2017) 1750131

Yildiz, A., see Mallick, S.

Yılmaz, S., see Mağden, A.

Yoon, D. W., Weighted minimal

6 (2017) 1750096

10 (2017) 1750140 translation

surfaces in

Minkowski 3-space

with density

Youssef, N. L. \&

Taha, E. H.,

Connections in

sub-Riemannian

geometry of

parallelizable

distributions

Yuksel, N., see

Karacan, M. K.

3 (2017) 1750039

5 (2017) 1750076

Z

Zaim, S.,

Guelmamene, H. \& Delenda, Y., Negative heat capacity for a Klein-Gordon oscillator in non-commutative complex phase space

10 (2017) 1750141

Zampini, A., see di Cosmo, F.

8 (2017) 1740005

Zhang, X.-H., see

Fang, X.-L.

9 (2017) 1750129

Zia, R., see Maurya,

D. C.

5 (2017) 1750077 